\title{
Identification and Measurement of Fibers in Scanning Electron Microscopy Images Using a High-Order Correlation Process
}

\author{
Oscar Yáñez-Suárez, Member, IEEE, and Mahmood R. Azimi-Sadjadi, Senior Member, IEEE
}

\begin{abstract}
This work describes the development of a dedicated system capable of identifying, measuring and counting various types of fibers and other objects in digitized scanning electron micrograph (SEM) imagery. The system uses a recursive high order correlation (HOC) process to extract the corner pixels of the fibers. The objects are defined by grouping connected corners, so that morphometric analysis can be performed. The method developed performs satisfactorily when the density of fibers per image ranges from low to medium. Simulation results for several cases are presented, along with a discussion on the capabilities and limitations of the current version of the system.
\end{abstract}

Index Terms - Fiberglass identification, image analysis, measurement technology.

\section{INTRODUCTION}

D IVERSE environmental, clinical, and quality assurance problems involve the evaluation of airborne particle distributions originated from materials and objects manufactured with fiberglass. This analysis is performed manually by observing scanning electron micrograph (SEM) imagery, finding an adequate observation field, recognizing the objects of interest, and measuring their geometrical properties using a reference grid or a pointing device. This task is tedious, error prone, and sensitive to observer's bias and eye-fatigue. It also requires specialized training and expertise in selecting the fields and picking the relevant objects in the scene. A dedicated digital image analysis system capable of detecting and measuring various types of fibers and other objects from SEM images under different operating conditions is therefore needed.

A number of conventional pattern recognition schemes may be applied to this problem [1]. However, in practice, they can only be partially successful when the density of fibers in an image is low. Other factors which may contribute to poor performance of these methods are: substantial variations in the sizes and shapes of the fibers, fibers can be crossing, very close together or be overlapping and/or obscured by other fibers, and large amounts of debris and other background objects present in the image. These, coupled with a number of other shortcomings such as sensitivity to different operating

Manuscript received July 20, 1998; revised January 11, 1999. This work was supported by Schuller, Inc., Denver, CO.

The authors are with the Department of Electrical Engineering, Colorado State University, Fort Collins, CO 80523 USA (e-mail: azimi@engr.colostate.edu).

Publisher Item Identifier S 0018-9456(99)02843-0. conditions and computational and speed limitations of the current pattern recognition systems, motivated this work to develop more efficient schemes for this problem. A dedicated system for identification, measurement, and classification of randomly scattered fibers in SEM images with low to medium fiber densities will be described in this paper. The development of such a system requires solving a number of technical issues ranging from the automated selection of observation fields to actual image analysis.

A sequence of different processing steps is followed for studying each image. A preprocessing stage consisting of thresholding and first-order differentiation is provided for achieving binary representation of the fiber edges. High-order spatial correlation (HOC) [2], [3] images are computed in each of four possible scanning directions. This procedure filters the fiber edge images by removing pixels that are not embedded in a consistent set of boundary pixels. The HOC-filtered images obtained are combined to detect pixels that are likely to be located in the corners of the fibers and other objects in the scene. Edge curvature analysis and edge association are finally used for the definition of individual objects. Section II below discusses the preprocessing scheme used for image segmentation. Section III describes the HOC process and how its results are applied for corner detection, selection and object identification. In Section IV, a discussion on the performance of the scheme for different typical cases is presented. Finally, Section $\mathrm{V}$ provides our concluding remarks.

\section{PREPROCESSING}

\section{A. Data Description}

The images studied in this paper are digital versions of SEM preparations of fiber glass obtained at $\times 1000$ magnification. They were digitized at $0.2 \mu \mathrm{m}$ per pixel, providing a total image size of $512 \times 512$ pixels. Gray-scale resolution was selected at four bits per pixel.

\section{B. Image Segmentation}

A fixed-level thresholding operator [4] was selected as the binarization algorithm based on the fact that most of the images used in this study exhibited a clearly bimodal gray-level distribution. The method performs nicely for those "clean" cases which do not contain debris and high clutter, but leads to some minor problems when applied to images 
with a high presence of debris and other nonfiber objects. The decision of keeping a fixed thresholding approach was made based upon its simplicity and the confidence that the analysis stages using HOC would be capable of rejecting most of the nonfiber information.

\section{Edge Detection}

In selecting the edge detection procedure the criterion was to use an algorithm that would yield fully connected edges for any fiber image. Classical edge detection algorithms usually produce noncontinuous traces to define the boundaries of objects [4]. These discontinuities would affect the performance of the subsequent HOC analysis. Given the fact that the image from which the edges are to be extracted is binary, a simple procedure can be used to obtain continuous border lines. Edge computation is performed by evaluating the firstorder differences between consecutive line scans of the image, and storing the resultant image for further processing. Four difference images have to be obtained to ensure that the edges will not have discontinuities. These images correspond to the row- and column-wise scans, both in the forward and backward directions, as follows [4]:

$$
\begin{aligned}
x_{r b}(m, n) & =x(m, n)-x(m-1, n), x_{r f}(m, n) \\
& =x(m+1, n)-x(m, n) \\
x_{c b}(m, n) & =x(m, n)-x(m, n-1), x_{c f}(m, n) \\
& =x(m, n+1)-x(m, n)
\end{aligned}
$$

where $x(m, n)$ is the image pixel at the $m$ th row and $n$th column. The resultant images will contain l's in every position where an edge has been detected. It is then straightforward to combine these difference images into the final edge-detected version, by unit thresholding followed by a logical OR operation, i.e.,

$$
\begin{aligned}
x_{\text {edges }}(m, n)= & g\left[x_{r b}(m, n)\right] \cup g\left[x_{r f}(m, n)\right] \\
& \cup g\left[x_{c b}(m, n)\right] \cup g\left[x_{c f}(m, n)\right]
\end{aligned}
$$

where $g[x]$ is a hard limiter thresholding function which is $g[x]=1$ for $x>0$ otherwise $g[x]=0$.

\section{FIBER ANALYSIS USING RECURSIVE High-ORDER CORRELATION}

\section{A. High-Order Correlation Process}

The main procedure used throughout the processing of the SEM images is referred to as the recursive "high-order correlation" (HOC) process. It provides a reliable mechanism to find and track sets of pixels that exhibit some consistent spatial pattern in a binary image. In this perspective, an image is viewed as a collection of sequential scan lines, moving either row-wise or column-wise.

A set of pixels, such as the one forming the edge of a fiber, forms a connected sequence across several of these scan lines. To identify a correlated pixel set, the spatial correlations among the points on three consecutive scan lines are formed, and the consistency in generating correlations in subsequent scans is determined. The process can be described by the following recursive equation [2]:

$$
\begin{aligned}
Y^{(k)}(i, n)= & g\left[\sum_{p \in W} \sum_{q \in W} Y^{(k-1)}(i, n) Y^{(k-1)}(i+p, n+1)\right. \\
& \left.\times Y^{(k-1)}(i+p+q, n+2)\right]
\end{aligned}
$$

where $k$ is the correlation order; $Y^{(0)}(i, n)=x(i, n)$ is the $i$ th pixel of the $n$th scan-line of the original image; $Y^{(k)}(i, n)$ is the result of the $k$ th-order correlation computation; $W=$ $\left\{m \mid m \in\left[-w_{s} / 2, w_{s} / 2\right]\right\}$ is the correlation window, relative to the $i$ th pixel; and $\left(w_{s}+1\right)$ is the correlation window size.

The size of the correlation window defines the shape of the region of support for the computation of the HOC and hence the kind of pixel patterns that are to be detected. For instance, a window size of one will produce nonzero correlations only for strictly horizontal or vertical pixel patterns. The correlation order $k$ defines the required length of the pixel sequences. For $k=1$, the term in the square bracket in (4) represents the correlation of pixels in three consecutive scan lines, i.e., $n, n+1$, and $n+2$ within the region of support determined by $W$. If there are at least three nonzero pixels in the relevant region, one per scan line, the HOC produces $Y^{(1)}(i, n)=1$ indicating a three-pixel spatial sequence. For $k=2$, the term in the square brackets represents the correlation of these three-pixel sequences, hence determining the correlations in a five-point sequence when $Y^{(2)}(i, n)=1$. Consequently, for a $k$ th-order HOC, $Y^{(k)}(i, n)=1$ represents consistent correlations in a $(2 k+1)$-pixel sequence.

Note that the choice of the order $k$ presents a tradeoff between the length of the pixel sequences detected and the sensitivity to pixel sequences representing segmentation noise. That is, increasing the order obviously increases the correlation length, but it also reduces the robustness to reject pixel sequences that are not located along the boundaries of fibers. This may result in wrong or missing object detections. The HOC process of second order was empirically found to perform optimally for our application. Fig. 1 shows examples of the HOC of first and second order, with window size three, applied to an image section containing a fiber boundary.

The resulting image $Y^{(k)}(i, n)$, referred to as the " $k$ th-order correlation image," is basically a filtered version of the original image, where only those pixels that lie within consistent pixel sequences are retained. This filtering action provides an excellent method for clutter or small debris rejection. As will be explained in the next section, the filtered images are also exploited to arrive at an image in which the fiber corners are identified. If the HOC calculations are performed twice for each pixel, both in the forward scan direction (4) and in the backward scan direction [obtained by replacing $n+1$ and $n+2$ by $n-1$ and $n-2$ in (4), respectively], the occurrence of a nonzero pixel in the same location for both scans allows for an even larger, $(4 k+1)$-pixel sequence to be detected.

Embedded in the structure of the HOC computation is the curvature and angle description of each segment [2]. The 

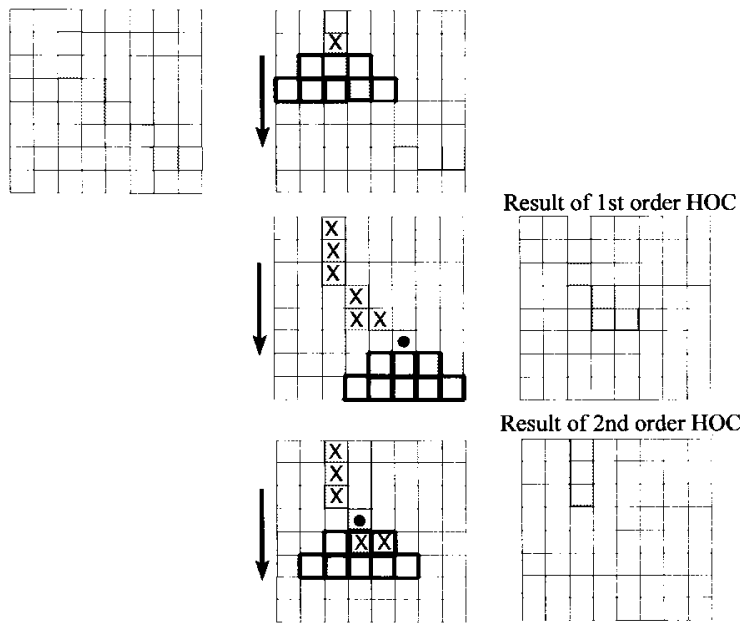

Fig. 1. Computation of the first and second order HOC for a fiber boundary segment, using a window size of three pixels. The pixels marked with a dot are the first ones in the row-wise scan to give zero-valued HOC.

three pixels, one from each consecutive scan line, used for computing a term of the HOC equation, define a pair of line segments. The angle between these two segments is the "local" curvature of the segment given by

$$
\mu=\tan ^{-1}\left(\frac{1}{q}\right)-\tan ^{-1}\left(\frac{1}{p}\right)
$$

where $p$ and $q$ are the indices of the pixels within each of the correlation windows defined in the HOC (4). In this way, a complete angle description of any given segment is obtained. The angle information is useful for discriminating various types of segments depending on their overall curvature. Additionally, the angle history of the segments can be used to differentiate the boundaries of straight-line fibers from those of curved ones and other objects or debris.

Taking into account that the images used for analysis (edges) are actually highly sparse matrices (for the sample images considered the pixel densities range from $10 \%$ to $35 \%$ ), a sparse matrix representation [5] of the edge image economizes memory usage and processing time, while allowing for the full vectorization of the HOC algorithm and an improved efficiency of the implementation. Another benefit is that the sparse matrix representation keeps track of the pixel location information, so the retrieval of corner coordinates can be performed without additional passes over the image.

To collect the basic information for the analysis procedure, the HOC is computed for the edge image in four different scanning directions, thus producing four HOC images. These images are then combined together to obtain a list of pixel coordinates that might represent fiber corners. Corners are finally associated, and the fiber count and measurements are obtained. These steps are described in the following sections.

\section{B. Corner Detection}

A HOC image of the fiber edges contains a considerable amount of information, describing all the available linear paths. Nevertheless, many of the remaining pixels are of little use for the purpose of identifying the fibers. This is due to the fact that most of these pixels lie on linear segments of the fiber edges, and they do not possess sufficient information for either locating or identifying the fibers. The same is not true for those pixels near or at the corners of a fiber. The corner information is valuable in locating the fibers and performing measurements on them. Every corner relates a pair of fiber edges, thus providing structural information that is required to group the edges into well-defined fibers. Consequently, locating the possible corner pixels results in an attractive option for data reduction and overall processing speed gains.

A set of HOC images can be utilized to extract the coordinates of those pixels close to or at the corners of the fibers. When scanning in any given direction, the filtering action of the HOC computation will actually remove those pixels that are found close to or at the corners of the fibers oriented in that scanning direction. Corner pixels oriented in the row-wise direction will remain only in one of the row-wise HOC images. If these images are combined using the XOR operation, the corner candidates will be retrieved. The same is true for the column-wise oriented corners. In this way, four HOC scan results are necessary to detect all the possible corner pixels. The corner detection operation can therefore be described by the following expression:

$$
Y_{\text {corners }}^{(k)}=\left[Y_{r f}^{(k)} \oplus Y_{r b}^{(k)}\right] \cup\left[Y_{c f}^{(k)} \oplus Y_{c b}^{(k)}\right]
$$

where $Y_{r f}, Y_{r b}, Y_{c f}, Y_{c b}$ are the four HOC images obtained for row-wise forward, row-wise backward, column-wise forward, and column-wise backward scanning, respectively. The union operation in (6) mixes the row-wise XOR'ed and column-wise XOR'ed images to collect all the possible corners without duplicating them. The nonzero pixels after this operation are the corner candidates.

For a given size of the correlation window and for a given order, the number of candidate pixels near any corner may vary widely. The resulting pixels will be clustered around each of the fibers' corners, forming sets from which the actual corners have to be selected in the subsequent processing steps. Also, the combination of window length and correlation order determines the amount of noise rejection in the filtering process. It is known that the increase in the window size when performing the HOC generally reduces the noise on the output images, while increasing the computation time [2]. Additionally, increasing the window size over five or seven pixels produces no significant changes to the output information. Increasing the order of correlation also impacts the noise rejection ability of the process, but produces very large sets of possible corner pixels. In the present application, a second-order HOC process with window size of five pixels was used for image filtering and pixel reduction, balancing a compromise between computation time and detection accuracy.

\section{Corner Selection}

Once all the potential corners are detected, the next step is to eliminate any false detections along the fiber sides. The local curvature information in (4) is used at this point to perform the refinement task. Only the pixels selected as candidates or potential corners in the previous processing step are considered for this analysis, effectively performing an additional amount 


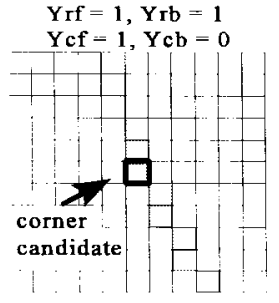

(a)

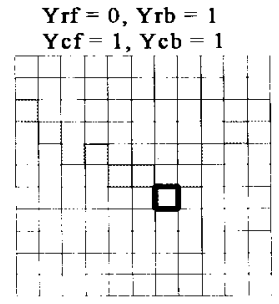

(b)

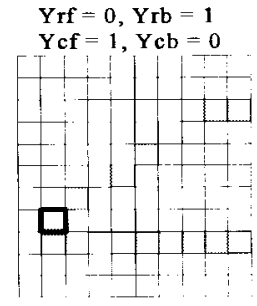

(c)
Fig. 2. Possible cases for corner selection and their acceptance status.

of data reduction. A corner candidate can occur given one of two possible conditions: either one of the row-wise or columnwise HOC images was zero for that pixel, as in Fig. 2(a) and (b), or one image of each scan direction (row- or column-wise) was zero, as in Fig. 2(c). Each case is treated separately when refining the corner pixel set.

Corner candidates with a single zero-valued HOC component are usually true corner pixels, but there may exist some pixels, found as part of a linear segment of fiber boundary, that may also be detected as possible corners because of the jagged appearance of the digitized linear segment. To distinguish between these two cases, the $4 k-1$ curvature angles, with $k$ being the correlation order, measured along the forward-backward pixel sequence associated with a corner candidate pixel, are analyzed for consistency. Similar curvature angles along the pixel sequence indicate the presence of a linear segment, and thus the candidate can be rejected as not being a corner pixel. On the other hand, a sequence of regular curvature measures that breaks at the candidate pixel to continue in a new, also regular sequence, indicates that the candidate is indeed a corner. Fig. 2(a) and (b) show these two conditions, respectively.

When one of each scan direction (row- or column-wise) HOC components for a candidate pixel is zero, such pixels are most often close to a corner. Instead of accepting all of these pixels as actual corners, a proximity criterion is applied for their selection. Assuming that these pixels are close to corners, a measure of closeness that can be used is the count of scan lines that have nonzero pixels within the HOC region of support. It was found adequate to decide for a corner pixel when this scan line count was at most one. Fig. 2(c) shows an example of this condition.

The set of selected corners is scanned for redundancies, i.e., for pixels that are too close to each other to be considered as separate corners. A nearest-neighbor clustering procedure removes these redundancies. A drawback of this procedure is that corners of some narrow fibers might be merged together, and the final object identification stage might miss the fiber.

\section{Data Association and Fiber Measurement}

With the corner pixels selected, the identification of fibers can be accomplished by performing a search and association pass over the image. For each selected corner, a Freeman chain-linking procedure [4] is performed over the edge pixels in the image, starting from the current selected corner. The chain-linking procedure allows for systematically and recursively visiting the eight-connected neighbors of a pixel, in order to track a connected curve. All the pixels in the current chain are tagged equally in order to identify them as being part of the same object. The chain is built until it reaches back to the starting point, or until there are no further pixels to add to the current chain. In this case, when the chain is not closed, the corner pixel from which the chain started is rejected as being an actual object corner. This corner elimination provides an additional level of noise rejection.

A closed chain represents an actual object in the scene. All the corner pixels that have previously been selected for that object will be included in the chain. Therefore, when the chain-building process is finished, all the selected corners for a given object share the same tag. In this way, the corners are associated and the object is completely identified. The chainbuilding procedure is repeated for the next nontagged corner pixel, assigning a new tag to each different chain found, until all the selected corners are tagged.

All the information for measuring the fibers and other objects in the image is now available. The data association pass generates sets of pixels that define the corners of an object/fiber. These corners form a unique irregular convex polygon (i.e., its convex hull [5]) that linearly approximates the edges of such object, and from which geometric measurements such as width and length can be taken. The ideal case is that all the polygons associated with fibers are four-sided, but this is not necessarily the case. Narrow fibers can be sometimes identified with only two corners, making the width information unavailable. Regular-sized fibers, that have a rounded, rather than sharp, corner can be identified with three pixels instead of four, so the width and length have to be inferred from the associated triangle. Sets of more than four corners can be related either to a single fiber with a rounded or curved boundary, multiple crossing/overlapping fibers, or an object of unspecified shape. These cases have to be treated in a different way. Although the basic identification of multiple crossing and/or overlapping objects is included in the current version of the analysis software, the issue of correctly identifying them is yet to be addressed. When the length and width of the identified objects are available, a simple aspect ratio check can be performed to classify the objects as either fibers or other generic particles. Objects with aspect ratios of $3: 1$ or higher are considered to be fibers. For objects identified with only two corners (narrow objects) the aspect ratio check is done considering a width equal to the length of the HOC path.

\section{Test Results}

Twenty-seven SEM images were analyzed in this study. These images varied in fiber density and amount of debris present. For analysis purposes, the images were visually classified in terms of their fiber density into one of two different cases, which are described in the following sections. It is important to note that exactly the same processing steps were applied to every image, regardless of its fiber density.

\section{A. Case 1-Low Fiber Density}

A typical sample of this category is shown in Fig. 3. This is a very clean image, with little amount of debris present. Because of the good contrast and low background noise, 

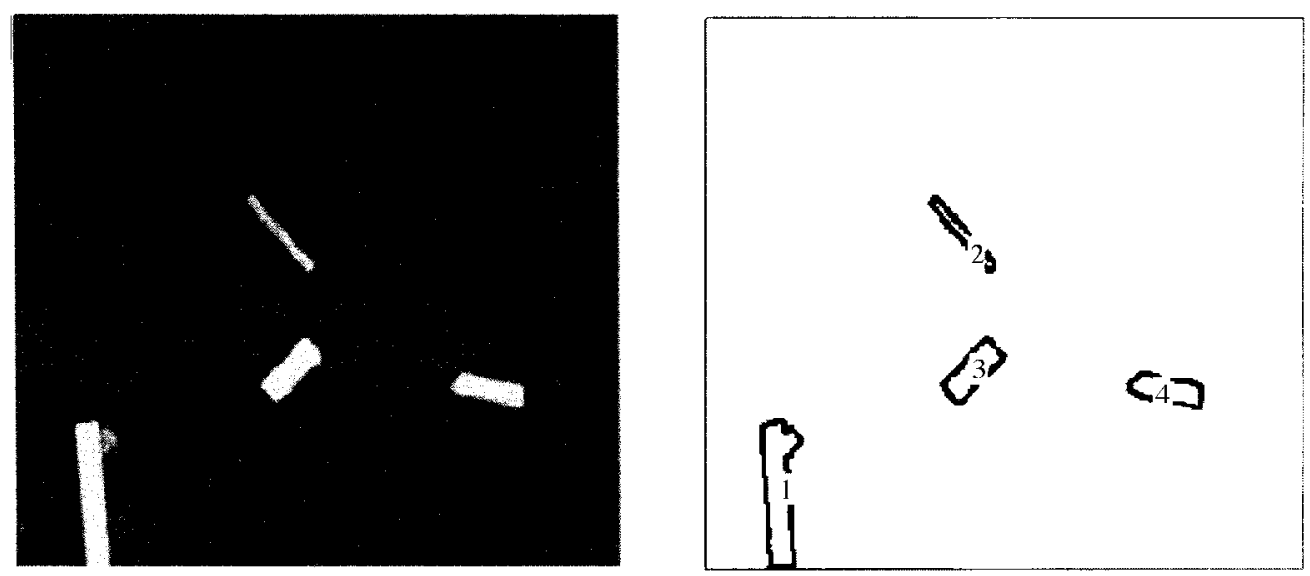

Fig. 3. Low-density SEM. Original image and final image showing the different objects detected.
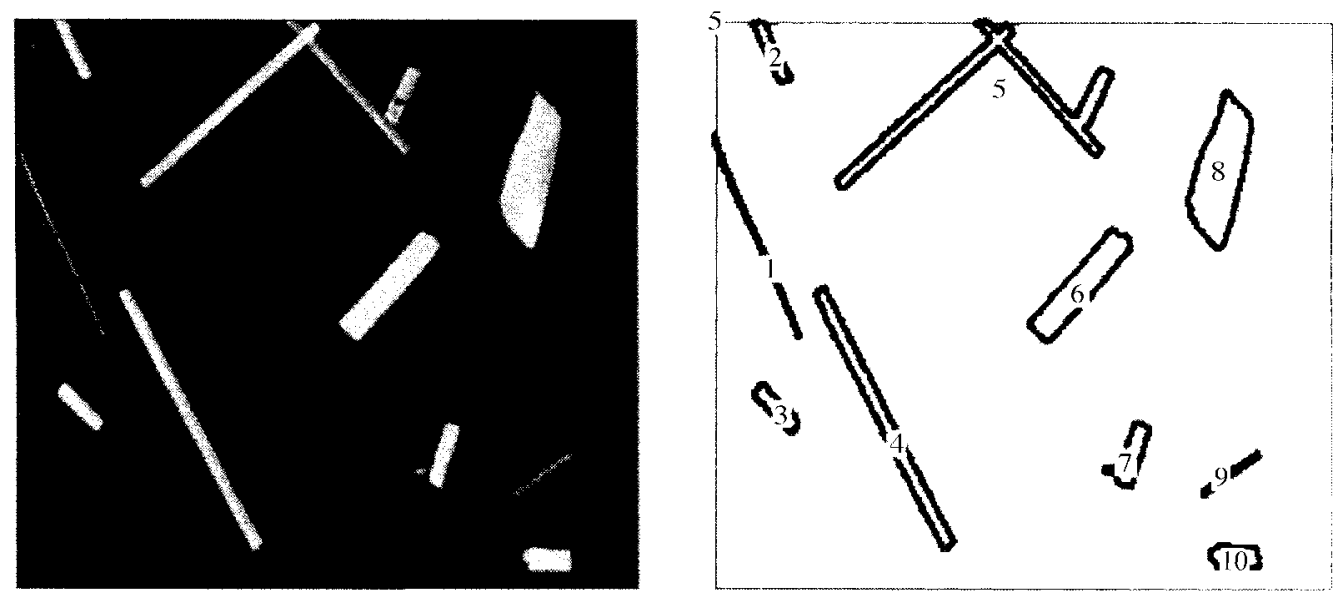

Fig. 4. Medium-high density SEM. Original image and final image showing the different objects detected.

the fixed thresholding segmentation performed adequately in detecting the individual objects. The corner selection and fiber identification procedures yielded complete information of the scene, and most of the fibers were correctly identified and measured, as shown in Table I(a). The small portion of debris next to fiber 01 is not detected as an individual object, but is merged into the polygon defining the fiber, basically because it is located too close to the actual fiber corner. It turns out that the impact of this on the fiber width measurement is significant. This is due to the fact that the fiber measurements are actually made based on the averages of the lengths of opposite sides of the polygons enclosing the fibers. Since one of the short sides is erroneously extended with the merged debris, the decision based on the aspect ratio classifies this object as a particle instead of as a fiber.

\section{B. Case 2-Medium-High Fiber Density}

Medium-high density cases, an example of which is shown in Fig. 4 and Table I(b), basically present the same behavior as the low density ones, except for the appearance of overlapping or crossing fibers. In the current implementation, crossing and overlapping fibers are detected as a single object, but no measurements are provided as they would be meaningless.
Again, the good contrast and low background noise allow for better identification of all the objects in the scene.

Fig. 5/Table I(c) and Fig. 6/Table I(d) show additional examples of the medium-high category. These images demonstrate that the system cannot handle correctly all the narrow fiber cases. In Fig. 5, due to the fixed thresholding segmentation approach, narrow fibers with poor contrast are lost in the edge detection phase. Further, this fixed thresholding is partly responsible for the cases where very close, parallel fibers are erroneously segmented as a single object. In Fig. 6, a narrow fiber is missed during the corner selection phase, because only one corner is selected for it (objects with just one corner selected are discarded). This is a consequence of the similarities of the correlation paths associated with segmentation noise and those that result from fiber edges being too close to each other as in the narrow fiber cases. In both situations, multiple correlation paths can be generated. Since the corner selection algorithm is tuned to reject multiple, randomly oriented paths, it is possible that an actual narrow fiber corner result is rejected. It has been described that there are several provisions for noise rejection throughout different processing stages, thus increasing the chances that the narrow fibers will be misdetected. 



Fig. 5. Medium-high density SEM. Original image and final image showing the different objects detected.
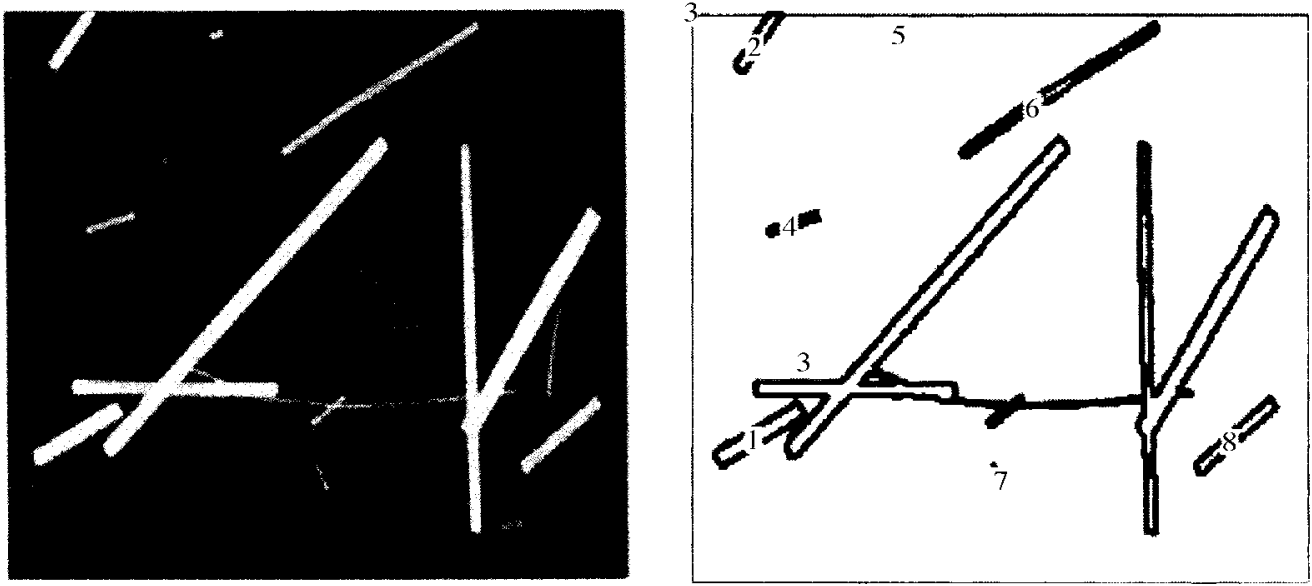

Fig. 6. Medium-high density SEM. Original image and final image showing the different objects detected.

\section{Limitations}

The use of a second-order HOC with window size of five pixels proved to be effective in reducing the segmentation noise in all but highly cluttered cases. Even for low density cases like the one shown in Fig. 3, the highly nonhomogeneous and cluttered background yields a lot of isolated "edges" that in some instances are long or close enough to produce nonzero correlations over the entire HOC region of support. While the candidate corner detection process rules out many of these noisy sections, the resultant computational overhead in the fiber identification process is inevitable. On the other hand, the HOC parameters selected produce paths that are too short for detecting corners in the cases where smooth, rounded edges, rather than sharp, well-defined turns are present. In these cases, the curvature analysis algorithms cannot detect the corners adequately, or they may even miss them completely.

The occurrence of segmentation noise is mainly due to the binarization method being used. It has been shown that fixed thresholding yields poor results when high-clutter or poorcontrast cases are processed. An alternative to this method is being developed where both the first (gradient) and the second (Laplacian) local derivative information is used to directly find the edges of the fibers and other relevant objects in the scene, thus avoiding any binarization step. A better edge detection algorithm will improve the chances of adequately segmenting narrow fibers, both by avoiding the low-contrast problems and allowing for more relaxed corner selection criteria as a result of the reduced segmentation noise.

The identification of composite objects that correspond to crossing or overlapping fibers has been successfully implemented in the data association pass. Within this process, the convex hull of each set of corners that correspond to the same object is computed. If all the corners are part of the hull, then the fiber is clearly enclosed in a polygon for which the length and width measurements can be performed. On the other hand, if some of the corners lie inside the hull, then the most probable situation is that this set of corners is describing a composite object. In the current implementation, if the number of corners lying inside the hull is less than four, then the object is considered a single fiber. This introduces some measurement error, as was described before for the case of object 01 in Fig. 3 . 
TABLE I

Objects Identified and Associated Measurements for SEM's in Figs. 3-6

\begin{tabular}{llll}
\hline Object Type & ID & Length & Width \\
Particle & 01 & 107.56 & 96.52 \\
Fiber & 02 & 83.93 & 9.22 \\
Fiber & 03 & 59.68 & 18.44 \\
Eiber & 04 & 68.73 & 17.12
\end{tabular}

(a)

\begin{tabular}{lcll}
\hline Object Type & ID & Length & Width \\
Narrow fiber & 01 & 196.33 & 0.00 \\
Fiber & 02 & 58.05 & 12.16 \\
Fiber & 03 & 48.27 & 11.18 \\
Fiber & 04 & 264.54 & 12.11 \\
Multiple & 05 & 0.00 & 0.00 \\
Fiber & 06 & 120.21 & 22.20 \\
& $\vdots$ & $:$ & $:$ \\
\hline
\end{tabular}

(b)

\begin{tabular}{llll}
\hline Object Type & ID & Length & Width \\
Narrow & 01 & 26.08 & 0.00 \\
Multiple & 02 & 0.00 & 0.00 \\
Narrow fiber & 03 & 72.20 & 0.00 \\
Fiber & 04 & 344.55 & 80.66 \\
Fiber & 05 & 159.36 & 10.03 \\
Narrow fiber & 06 & 56.92 & 0.00 \\
Fiber & 07 & 139.32 & 20.59 \\
Fiber & 08 & 59.62 & 1928 \\
\hline
\end{tabular}

(c)

\begin{tabular}{llll}
\hline Object Type & ID & Length & Width \\
Fiber & 01 & 99.51 & 14.11 \\
Fiber & 02 & 61.08 & 11.83 \\
Multiple & 03 & 0.00 & 0.00 \\
Fiber & 04 & 50.83 & 6.66 \\
Particle & 05 & 11.40 & 6.00 \\
Fiber & 06 & 189.55 & 51.76 \\
Narrow & 07 & 22.85 & 0.00 \\
Fiber & 08 & 99.87 & 9.12 \\
\hline
\end{tabular}

(d)

\section{CONCLUSION}

An approach to the identification and measurement of fiberglass particles in SEM imagery has been presented. Through the processing of the segmented images using HOC filters in four different scan directions, almost perfect detection of the relevant objects in the scene is achieved. Discrimination among the different types of detected objects is performed based on basic geometrical measurements. The scheme performs adequately when a low fiber density is observed. For higher density cases, although the method is able to discriminate composite objects in the scene, additional provisions have yet to be incorporated for an improved characterization. The approach is well suited for on-line applications, as the computations can be realized in a parallel fashion, so that machine limitations like memory swapping or input/output, are accounted more for delays than the actual HOC computations or the data association process.

\section{REFERENCES}

[1] R. O. Duda and P. E. Hart, Pattern Classification and Scene Analysis. New York: Wiley, 1973

[2] R. J. Liou and M. R. Azimi-Sadjadi, "Dim target detection using high order correlation method," IEEE Tran. Aerosp. Electron. Syst., vol. 29, pp. 841-856, July 1993

[3] R. J. Liou and M. R. Azimi-Sadjadi, "Multiple target detection using modified high order correlation," IEEE Trans. Aerosp. Electron. Syst., vol. 34, pp. 553-568, Apr. 1998.

[4] A. K. Jain, Fundamentals of Digital Image Processing. Englewood Cliffs, NJ: Prentice-Hall, 1989.

[5] J. Sedgewick, Algorithms. Englewood Cliffs, NJ: Prentice-Hall, 1988

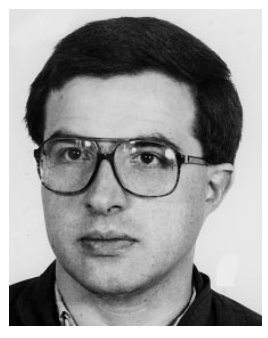

Oscar Yáñez-Suárez (S'92-M'97) received the B.S. and M.Sc. degrees in biomedical engineering from the Universidad Autónoma MetropolitanaIztapalapa, México, in 1988 and 1993, respectively. $\mathrm{He}$ is currently pursuing the Ph.D. degree in electrical engineering at Colorado State University, Fort Collins.

His research interests include pattern recognition for computer-aided medical diagnosis, structurally adaptive neural networks, and biomedical signal and image processing.

Mr. Yáñez-Suárez is a Member of the IFMBE and the Mexican Society of Biomedical Engineering.

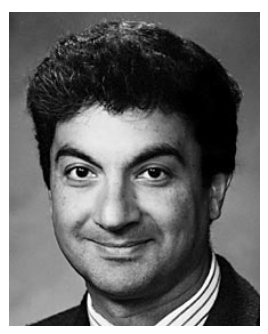

Mahmood R. Azimi-Sadjadi (S'81-M'89-SM'91) received the M.S. and Ph.D. degrees in electrical engineering with specialization in digital signal/image processing from the Imperial College of Science and Technology, University of London, London, U.K., in 1978 and 1982, respectively.

$\mathrm{He}$ is currently a Full Professor, Electrical Engineering Department, Colorado State University (CSU), Fort Collins. He also serves as the Director, Multisensory Computing Laboratory, CSU. His main areas of interest include digital signal and image processing, target detection, classification and tracking, adaptive filtering and system identification, and neural networks. His research efforts in these areas resulted in more than 100 journal and refereed conference publications. He is the coauthor of the book Digital Filtering in One and Two Dimensions (New York: Plenum, 1989).

Dr. Azimi-Sadjadi was the recipient of the 1993 ASEE-Navy Senior Faculty Fellowship Award, 1991 CSU Dean's Council Award, and 1984 DOW Chemical Outstanding Young Faculty Award. He is an Associate Editor of the IEEE Transactions on Signal Processing. 